\title{
A Thermodynamic Approach to Predict Dopant Atoms Segregation on Nanocrystals
}

\author{
Daniel G. Stroppa*,**, Luciano A. Montoro*, Antonio Campello***, Armando Beltrán****, \\ Juan Andrés****, Edson R. Leite*****, Antonio J. Ramirez* \\ * Brazilian Synchrotron Light Laboratory, Campinas, SP, Brazil. antonio.ramirez@lnls.br \\ ** Mechanical Engineering School, University of Campinas, Campinas, SP, Brazil \\ *** Institute of Mathematics, Statistics and Scientific Computing, University of Campinas, Campinas, \\ SP, Brazil \\ **** Departament de Química Física i Analítica, Universitat Jaume I, Castellón de la Plana, Spain. \\ ***** Department of Chemistry, Federal University of São Carlos, São Carlos, SP, Brazil
}

The development of novel nanostructured devices requires reliable modeling procedures for the description of materials features at the atomic scale. Among the most significant challenges in this field is the prediction of nanocrystals morphology and growth behavior as function of synthesis parameters from first principles.

This work describes a novel model for the evaluation of dopant segregation on faceted nanocrystals from the ab initio calculated surface energy values as function of dopant content for different crystalline planes [1]. Starting from an arbitrary nanocrystal geometrical configuration, an iterative calculation procedure for incremental dopant contents is performed to verify the most likely dopant segregation state as a function of the nanocrystal overall composition. The selection rule applied at each iteration step was the minimization of the Wulff parameter deviation for each crystalline plane. The Wulff parameter for a given crystalline plane is equal to its surface energy density divided by its respective facet distance.

The expected dopant segregation patterns for two geometrical configurations were compared to semi-empirical characterization results from $\mathrm{Sb}$-doped $(4.5 \%$ atom, $13.9 \%$ atom $) \mathrm{SnO}_{2}$ nanocrystals obtained by a nonaqueous synthesis route [2]. The comparison between the measured and the calculated dopant atoms distributions on the nanocrystals facets are in a good agreement, with a mean deviation value below $5 \%$.

The successful description of the dopant atoms segregation by this model indicates that first principles calculations can be used to predict nanocrystals features at thermodynamic equilibrium configurations, which is a feasible approximation for a number of experimental systems. In addition, the use of Wulff parameter deviation as the main selection rule points out that the real nanocrystalline systems tend to use dopant diffusion for the minimization of the overall surface energy as the growth processes take place [3].

\section{References}

[1] Beltrán, A et al.; Appl. Phys. Lett., 83 (2003), 635-637.

[2] Stroppa, D. G. et al; J. Am. Chem. Soc., 131 (2009), 14544 - 14548. 

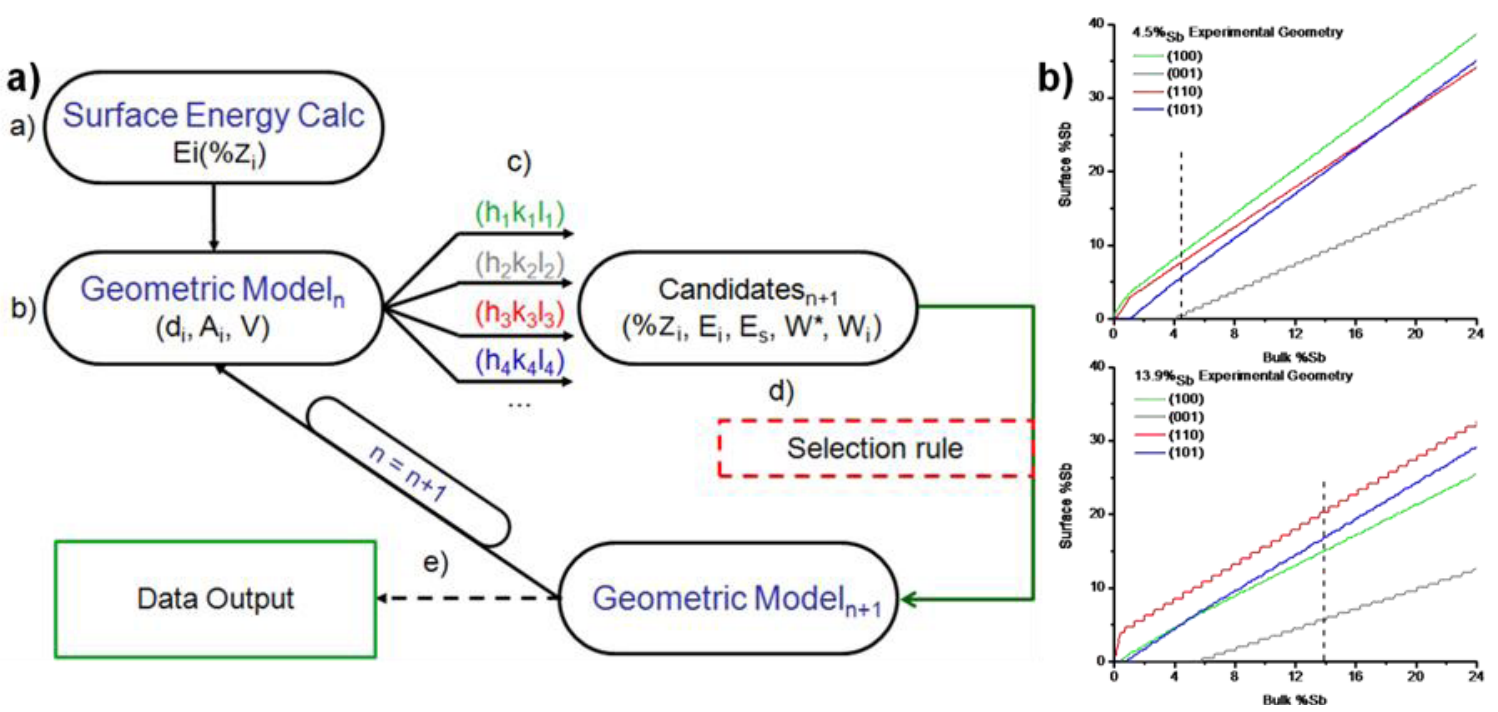

Figure 1: a) Flowchart of proposed model implementation and b) calculation results for the $4.5 \% \mathrm{sb}$ and $13.9 \% \mathrm{sb}$ experimental geometries. The dashed lines indicates the experimentally measured dopant overall contents, respectively, and indicates the expected dopant distribution for thermodynamic equilibrium.

Table 1: Evaluation of surface energy and Sb content for the proposed ATO nanocrystal models.

\begin{tabular}{lcccccc}
\hline \multicolumn{7}{c}{ ATO 4.5\% Sb Geometry - 120 dopant atoms } \\
\hline Facet & Distance (nm) & Surface Area (nm2) & Energy (J/m2) & Expected Content (\%) & Atoms & Reference [2] \\
\hline$[\mathbf{1 0 0}]$ & 2.85 & 19.47 & 3.78 & 8.5 & 29 & 28 \\
{$[\mathbf{0 0 1}]$} & 2.40 & 11.32 & 3.13 & 0.5 & 1 & 0 \\
{$[\mathbf{1 1 0}]$} & 2.69 & 34.42 & 3.45 & 7.4 & 45 & 48 \\
{$[\mathbf{1 0 1}]$} & 2.65 & 47.41 & 3.44 & 5.4 & 45 & 44 \\
\hline \hline \multicolumn{7}{c}{ ATO 13.9\% Sb Geometry - 172 dopant atoms } \\
\hline Facet & Distance (nm) & Surface Area (nm2) & Energy (J/m2) & Expected Content (\%) & Atoms & Reference [2] \\
{$[\mathbf{1 0 0}]$} & 1.90 & 29.21 & 5.18 & 14.8 & 76 & 87 \\
{$[\mathbf{0 0 1}]$} & 1.76 & 11.31 & 4.65 & 5.5 & 11 & 10 \\
{$[\mathbf{1 1 0}]$} & 2.35 & 7.32 & 6.87 & 20.3 & 26 & 15 \\
{$[\mathbf{1 0 1}]$} & 2.12 & 20.16 & 5.54 & 16.7 & 59 & 60 \\
\hline
\end{tabular}

Keywords: Dopant Distribution, Surface Energy ab initio Calculation.

Acknowledgements: The authors acknowledge the financial support of the Brazilian research funding agencies FAPESP, CNPq, and FINEP. 